\title{
Three-dimensional Analysis of an In Situ Double-torsion Test by X-ray Computed Tomography and Digital Volume Correlation
}

\author{
P. Leplay $\cdot$ J. Réthoré $\cdot$ S. Meille $\cdot$ M.-C. Baietto $\cdot$ \\ J. Adrien · J. Chevalier · E. Maire
}

\begin{abstract}
The double-torsion (DT) test is commonly used to characterize the slow crack growth behavior of brittle materials. However, it relies on several mechanical hypotheses which still need to be deeply tackled. Measuring the full 3D displacement field experimentally through in situ experiments would improve the understanding of the mechanical behaviour. A dedicated experimental set-up was designed to perform in situ tests in a X-ray Computed Tomography (XCT) scanner on a porous brittle ceramic which is optically opaque. The analysis of the three-dimensional images with Digital Volume Correlation (DVC) allows a first study of the global kinematics and the stable crack growth propagation. This experimental and numerical methodology presents a strong potential for a future deep understanding of this complex mechanical test.
\end{abstract}

Keywords Double-torsion test $\cdot$ X-ray computed tomography $\cdot$ Digital volume correlation $\cdot$ Slow crack growth $\cdot$ Brittle materials

P. Leplay $(\bowtie) \cdot$ J. Réthoré $\cdot$ M.-C. Baietto

University of Lyon, CNRS INSA-Lyon, LaMCoS UMR 5259, 69621 Villeurbanne, France

e-mail: paul.leplay@saint-gobain.com

P. Leplay $\cdot$ S. Meille $\cdot$ J. Adrien · J. Chevalier · E. Maire University of Lyon, CNRS INSA-Lyon, MATEIS UMR 5510, 69621 Villeurbanne, France

P. Leplay

Saint-Gobain CREE, BP 224, 84306 Cavaillon, France

\section{Introduction}

\section{A Complex Mechanical Test: DT}

Many mechanical tests exist to characterize the fracture behavior of materials. Among the most widely used, the bending test is easy to setup and to analyse. The doubletorsion (DT) test, that can be seen as an extension of a bending test, is more specific to the ceramic materials. Indeed, it enables a stable crack propagation and the measurement of slow crack growth curves even for the most brittle materials like ceramics [1,2]. This test consists in bending one of the edges of a rectangular notched plate put on four rollers at each corner (Fig. 1). In such a setup, one face is loaded in tension whereas the other one is loaded in compression. After a pre-cracking procedure, the DT test can be used for the determination of the fracture toughness or the analysis of slow crack growth. The toughness measurement is conducted at very high loading rate and the record of the maximum load before failure and pre-crack size allows to obtain $K_{I C}$. Slow crack growth curves as a function of the mode $I$ stress intensity factor can be measured with a single specimen using different methods: either by a load relaxation test via a compliance calibration curve, or by loading the specimen at different loads and measuring the crack growth rates with an optical microscope [2]. Whatever the method used, the stress intensity factor $K_{I}$ is often considered in a first approximation as independent of crack length within the linear elastic fracture mechanics framework (equations (1) and (2) - Notations are given in Fig. 1):

$K_{I}=S_{m} P\left(\frac{3(1+v)}{\psi(S, t) S t^{4}}\right)^{1 / 2}$ 

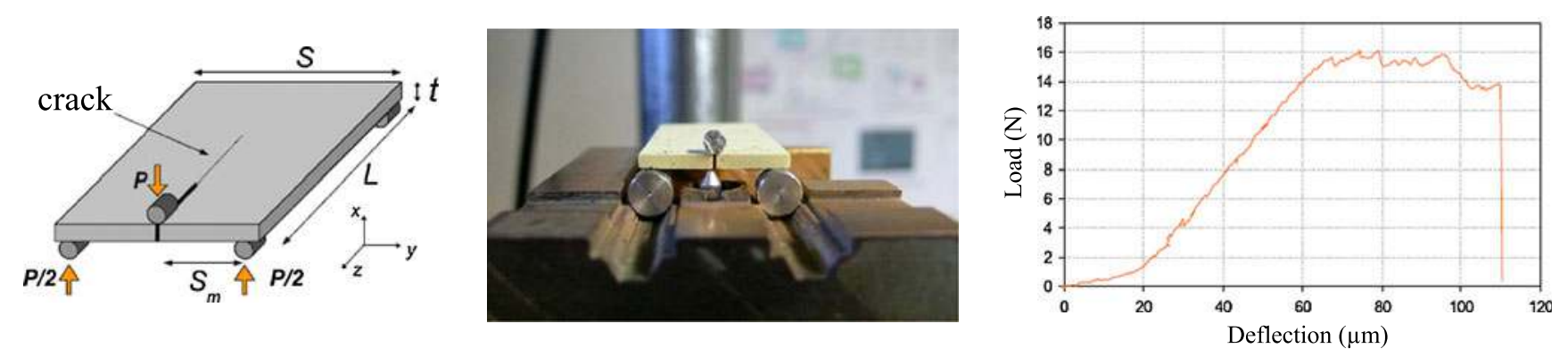

Fig. 1 Scheme, experimental double-torsion set-up and load-displacement response during test performed on silicon carbide sample

$\psi(S, t)=1-\frac{1.26 t}{S}+\frac{2.4 t}{S} \exp (-\pi S / 2 t)$

This independence of $K_{I}$ as a function of crack length explains why the crack propagation remains stable on a long distance despite the high brittleness of ceramics. This also explains why some mean propagation velocities $v$ can be measured depending on the applied stress intensity factor $K_{I}$. These $v-K_{I}$ diagrams include different regimes defined using threshold values, which is very useful to study the interaction between the crack and its environment [3, 4].

Introduced at the end of the 1960s [5], the DT test has been made popular by the different studies of Evans [1, 6]. Then its use has been widened to characterize at high temperature the fracture behavior of numerous ceramics like alumina [7], mullite [4], zirconia [8], cordierite [9] and natural rocks [10]. For instance, the DT test has been used to study the cracking behavior of porous ceramics for Diesel particulate filter applications [11]. This test has already been the subject of several literature reviews these last decades $[2,12,13]$.

Beyond its wide use in literature, the DT test presents some questionings. Indeed, the stress intensity factor $K_{I}$ calculation relies, through equation (1), on several mechanical hypotheses:

- Once the crack has started to propagate, the specimen is considered as two separate halves, loaded in torsion, without any interaction or contact between them. It is also supposed that the uncracked part of the sample remains undeformable [1].

- The stress intensity factor calculation may depends on the specimen size and the loading conditions... Numerous corrections factors were proposed to address this point [10].

- The mechanical behavior of the material is supposed to be perfectly linear elastic. In some cases, high temperature loading or specific material, a non-linearity may arise. In such a case, the toughness calculation is invalid [14].

- The front shape is supposed to be straight for $K_{I}$ calculation. It was observed that this crack front is curvilinear, longer along the tensile face of the specimen than along the compressive face $[15,16]$. Since the exact crack front shape will influence the stress intensity factor distribution, some additional correction factors must be used to estimate the local crack propagation velocity $[15,17]$.

- The stress intensity factor $K_{I}$ value is independent of the crack length (equation (1)). These assumptions are satisfied over only a finite range of crack lengths. Several ranges have been proposed [18-20].

These different limitations and questionings are partly responsible for the fact that the DT test has never been successfully standardized. Few numerical studies based on finite element simulations have been performed to tackle these difficult questions [10, 14, 18, 19]. Recently [10], a three-dimensional finite element analysis of a very large grooved specimen was carried out. Empirical corrective factors of the classical equation were proposed to account for the influence of the groove geometry and the crack length values on the strain energy rate for specimens, illustrating the fact that this equation is generally inadequate. But no general conclusions can be obtained. A very interesting paper has recently used DT to study by stereo Digital Image Correlation in 2D the crack growth and the fracture process zone formation for two nuclear grade graphites [37].

To improve the understanding and the use of this test, different requirements must be met simultaneously: the determination of the actual displacement field within the loaded sample and the actual crack front shape evolution during the crack growth propagation. The quantification of these actual data is the first step to remove the simplifications made to propose an analytical approach of this DT test.

\section{Two Complementary Analysis Methods: XCT and DVC}

In the last decade, the development of X-ray tomography has led among others to the analysis of the bulk material structure or deformation [21]. The X-Ray Computed Tomography (XCT) principle consists in sending an X-ray beam onto a sample for $\mathrm{N}$ given angular positions, recording a series of $\mathrm{N}$ radiographies from which a $3 \mathrm{D}$ image is 
reconstructed. It offers an alternative to post-mortem analysis and techniques like microscopy which give also valuable information at the sample surfaces. It has also the advantage to allow a local analysis whereas stiffness or acoustic techniques give global data. The development of in situ experiments monitored by XCT has then led to a deeper understanding of the deformation mechanisms or of the structure behaviour during mechanical monotonic or cyclic loadings [22-25]. But to get a more quantitative analysis of those 3D data, one might use Digital Volume Correlation (DVC).

Introduced in the 1980s [26], Digital Image Correlation (DIC) is now widely used in experimental mechanics. Based on the optical flow equation, this full-field method enables to measure the displacement field of a structure from two images taken at different mechanical loadings. In $2 \mathrm{D}$, numerous softwares are currently available in laboratories and industries. In the present approach, the optical flow equation is solved by a global least squares method. This allows for choosing arbitrary basis functions for decomposing the displacement field over the area of interest. In a general case, finite element shape functions can be used [27]. In more specific cases (cracks [28, 29], beam [30], Brazilian test [31]), closed form analytical solutions are used to improved the robustness of the displacement estimation and to extract directly parameters that have a mechanical content (stress intensity factors e.g.). This type of closed form solutions is usually used to circumvent low signal over noise ratio, like it is encountered for instance in the case of ceramic material at ambient temperature [30] or at high temperature [32]. Some of these DIC techniques have been recently extended to DVC [22, 23, 33].

In this paper, a dedicated in situ DT device under XCT was developed and DVC techniques were used to quantify the 3D displacements and to follow the crack growth. The aim of this paper is not to be able now to check all the DT hypotheses above-listed, but to present an innovative way to capture essential 3D data for a future better understanding of this complex test. Section "Experimental Methods for
DT under XCT" details the development of the experimental set-up to perform in situ DT tests with a laboratory tomograph. Section "Application of DVC and First Results" presents the different results, the 3D displacement fields and the crack front evolutions successfully analysed thanks to DVC. Section "Conclusion and Prospects" concludes this study by proposing some different prospects.

\section{Experimental Methods for DT under XCT}

\section{Prototype Description}

An experimental set-up to carry out DT tests was specially designed to be mounted inside a laboratory tomograph [24, 34,35 ] and allows in situ quantitative analysis of deformation mechanisms. The sample is oriented vertically during the test to enable a full rotation during scan acquisition without any influence of the loading system on the 2D radiographies. The loading is carried out by a pliers that holds the specimen on its lower side, where a notch has been sawed (Fig. 2). Increasing loads are applied to the sample by means of a stepping motor through three cylindrical rollers. A minimum displacement rate on the sample of about $1 \mu \mathrm{m} / \mathrm{min}$ can be achieved. The outer surface of the XCT scanner beam gun has a controlled temperature of $23{ }^{\circ} \mathrm{C}$ to ensure the X-Ray tube thermal stability during the whole experiment [24]. The load applied on the sample is measured using a miniaturized load cell of $100 \mathrm{~N}$ (Fig. 2). The span between rollers is $8.5 \mathrm{~mm}$, the samples are parallelepipeds of $22 \times 2.1 \times 41 \mathrm{~mm}^{3}$. The notch is machined with a diamond saw of $500 \mu \mathrm{m}$ thickness, with a total length of approximately $10 \mathrm{~mm}$. Its notch tip radius is approximately $150 \mu \mathrm{m}$. Samples of a similar geometry have been tested also using a classical DT test, the sample being loaded horizontally [36]. Notched four-point bending tests have also been carried out on $14.4 \times 7.8 \times 150 \mathrm{~mm}^{3}$ bars with a initial notch $a_{0}$ of $3 \mathrm{~mm}$ to estimate the fracture toughness.
Fig. 2 Experimental device for DT with XCT. Sample dimensions $=22 \times 2.1 \times 41 \mathrm{~mm}^{3}$

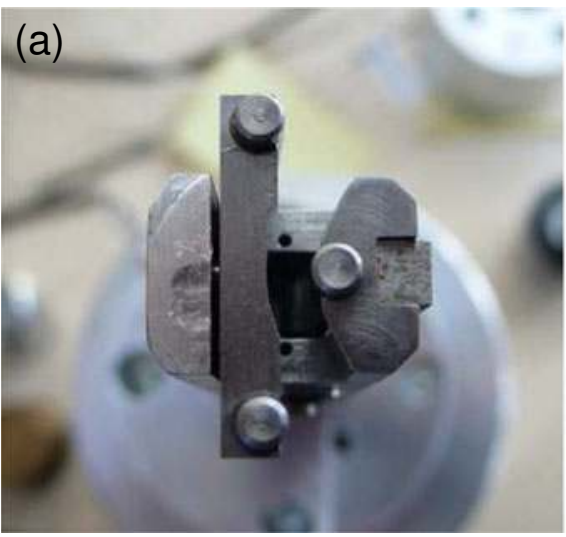

Zoomed top view without sample

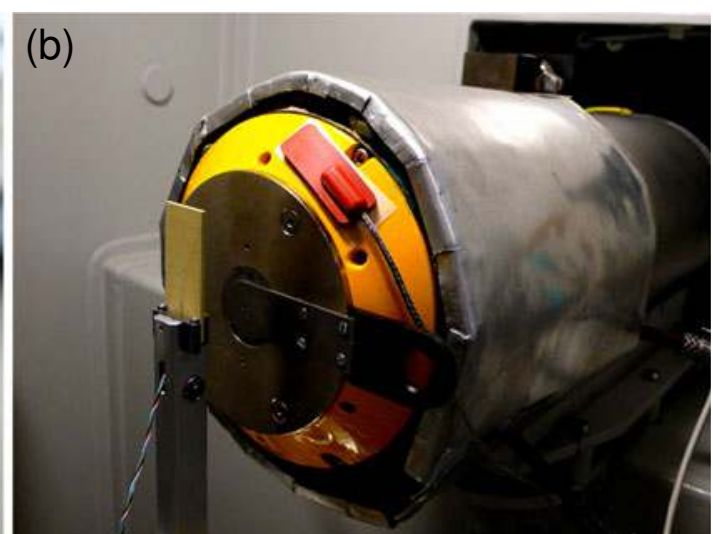

Enlarged view with sample 
The scanned volumes during in situ tests are located just above the notch to follow the crack propagation. The XCT scanner is a Phoenix X-ray v/Tome/xs. Changing the distance between the sample and the X-Ray gun allows to use several resolutions: from a resolution of $7.9 \mu \mathrm{m} / \mathrm{voxel}$, used to analyse the crack propagation over a scanned volume height of about $12 \mathrm{~mm}$, down to a resolution of $3.1 \mu \mathrm{m} /$ voxel used to obtain more precise scans for a scanned height of about $4 \mathrm{~mm}$. In this latter case, the scan is performed in the so called "local tomography" mode, i.e. the width of the sample being larger than the field of view [35]. The material tested is a recrystallized porous silicon carbide $\mathrm{R}-\mathrm{SiC}$ with a pore volume fraction of about $50 \%$ and a Young's modulus of about $60 \mathrm{GPa}$. It shows a purely elastic brittle behavior at room temperature.

\section{Experimental Procedure}

The experimental procedure starts with the acquisition of a reference volume at a small load of $0.5 \mathrm{~N}$, just enough to avoid any movement of the sample during rotation for the scan acquisition. Then the applied load is increased and additional scans are recorded, first in the linear elastic domain and then after crack propagation in the non linear domain. The acquisition of each scan lasts about 40 minutes. To avoid any crack propagation in the sample during the time necessary for data acquisition, the sample must be unloaded down to $65 \%$ of the maximal load before each scan. It has been confirmed by DT experiments that load relaxation can be noticed above $75 \%$ of the applied maximal load [2]. This unloading phase unfortunately reduces the relative displacement intensity in comparison to the initial reference scan and therefore affects the DVC analysis. The final validity of each in situ test is checked after the failure of the sample. The test is only declared valid if the crack has propagated straight through the specimen, vertically, with a tolerance angle of $\pm 10^{\circ}$.

\section{Tests Results, Validation of the Prototype}

A load-displacement of the stepping motor curve is shown in Fig. 3 of an in situ DT test. Eight different scans have been recorded during this test, to check the displacement and load proportionality within the elastic domain and to record several crack propagation steps. Four samples were tested. Three of them have failed correctly with a straight vertical crack. The maximum load recorded during the experiments was $16.0 \pm 0.4 \mathrm{~N}$, that leads to a critical stress intensity factor $K_{I}$ of $0.66 \pm 0.04 \mathrm{MPa} . \mathrm{m}^{1 / 2}$ using linear elastic fracture mechanics (equations (1) and (2)). This value is similar to the one measured using notched bending tests or standard double-torsion tests performed with the horizontal sample. The difference between the values

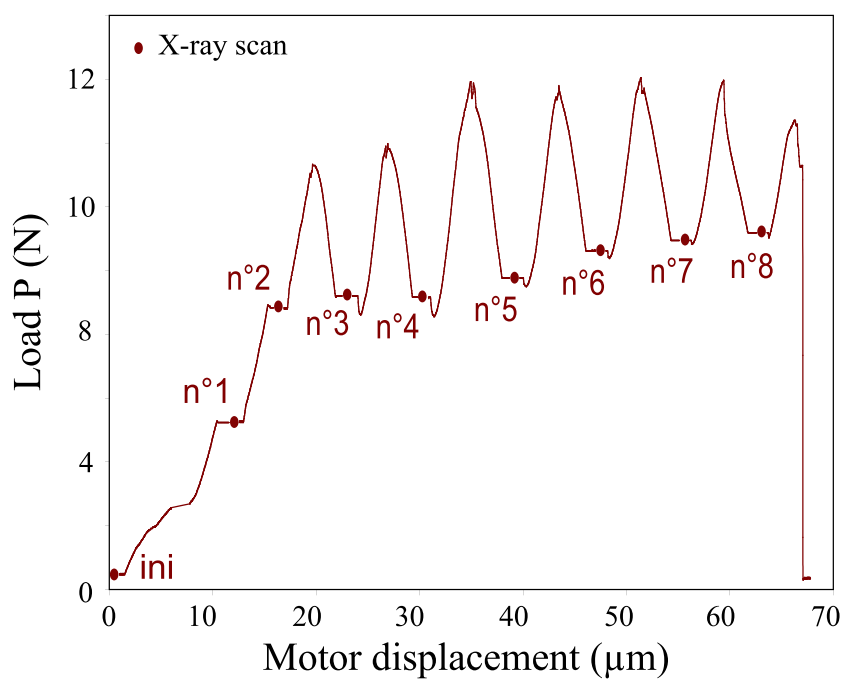

Fig. 3 Stepping motor displacement versus the measured load during the scans performed for the silicon carbide sample

obtained according to these different methods is lower than $7 \%$. The in situ double-torsion experimental set-up is thus validated.

\section{Application of DVC and First Results}

\section{Principle of DVC}

DVC is a full-field measurement method based on the analysis of two digital volumes containing grey level information for each voxel. Let us call $f$ and $g$ two scans corresponding respectively to a reference and a deformed state. Let us also call $\mathbf{u}$ the actual 3D displacement field of the structure. It is postulated that the texture of the sample microstructure is passive. Because of the grey level conservation principle, the evolution between $f$ and $g$ can only be attributed to the displacement field $\mathbf{u}$. The optical flow equation can be written for each voxel of coordinate $\mathbf{x}$ :

$f(\mathbf{x})=g(\mathbf{x}+\mathbf{u}(\mathbf{x}))$

The numerical problem which consists in estimating the displacement field is an ill-posed problem, even under the no-noise assumption. It is thus only possible to approximate the actual displacement field $\mathbf{u}$ by the best approximation possible called $\mathbf{v}$. The optical flow equation is written as a minimization of the global error over a chosen region $\Omega$ through a functional $\phi^{2} . \mathbf{v}$ is the displacement field solution best satisfying the optical flow equation thus minimizes:

$\phi^{2}(\mathbf{x})=\iiint_{\Omega}[f(\mathbf{x})-g(\mathbf{x}+\mathbf{v}(\mathbf{x}))]^{2} d \mathbf{x}$ 
Additional assumptions can be formulated to solve the problem. The displacement field $\mathbf{v}$ is decomposed over a set of analytical basis functions $\mathbf{N}_{i}(\mathbf{x})$ a priori selected. $\alpha_{i}$ are the associated degrees of freedom quantifying the contribution of the different basis functions $\mathbf{N}_{i}(\mathbf{x})$ through their respective amplitudes:

$\mathbf{v}(\mathbf{x})=\sum_{i} \mathbf{N}_{i}(\mathbf{x}) \alpha_{i}$

A non-linear least-square algorithm is then used to solve the minimization of equation (4). Then it is possible to calculate a posteriori the correlation error revealing where the optical flow has not been respected. From the solution $\mathbf{v}$ and the deformed volume $g$, a virtual reference volume is calculated and compared to the real one $f$. A correlation residual $R$ is obtained for each voxel $\mathbf{x}$ :

$R(\mathbf{x})=|g(\mathbf{x}+\mathbf{v}(\mathbf{x}))-f(\mathbf{x})|$

A low correlation value means a reliable displacement measurement using DVC.

The quality of the measurement depends also on the chosen shape functions $\mathbf{N}_{i}(\mathbf{x})$ in equation (5). Adding some appropriate kinematics hypothesis improves the DVC accuracy. Under two dimensional assumptions, Q4 bilinear finite element shape functions $\mathbf{N}_{i}(\mathbf{x})$ can be used to capture a continuous displacement field [27, 31]. Similarly under 3D assumptions, it is possible to use C8 trilinear shape functions [22, 23]. Compared to classical correlation approaches [33], the use of finite element shape functions to decompose the displacement field increases the measurement accuracy by assuming a continuous displacement from one element to another. Please note that more sophisticated approaches may be used based on shape functions dedicated to specific test kinematics [28-31].
Measurement Results of the 3D Displacements

Figure 4 represents the last scan ( ${ }^{\circ} 8$ shown in Fig. 3) obtained for one silicon carbide sample. It is already possible to distinguish the crack path on the sample face loaded in tension. To determine the exact crack tip location and to quantify the displacement field, the DVC method is applied to these scans, with $\mathrm{C} 8$ finite element shape functions.

All DIC methods need to have contrasted volumes in order to evaluate the displacement of the material. In 2D, a black pattern is usually added with a paint spray. Here in $3 \mathrm{D}$, the high porosity of the ceramic can be directly used as marker. Indeed, it shows a sufficient contrast since the mean pore size $(15 \mu \mathrm{m})$ is here about twice the size of a voxel.

The accuracy of the full-field method will also depend on the mesh size. When the elements are very small, strong local variations of the displacement field can be measured. This is useful especially near the crack. When the elements are very large, they contain a lot of grey level information and lead to low-noisy results (Fig. 5). In the present study, cubic elements of size of $16 \times 16 \times 16$ or $32 \times 32 \times 32$ voxels are the best compromise in terms of accuracy (Fig. 6).

Figure 7 represents the evolution of the kinematic fields at different steps of the loading for a silicon carbide sample. Only the view of the tensile face is shown on this figure. The measured displacements in the direction $y$, normal to the crack, are of the order of \pm 1 voxel. Being at a resolution of $7.9 \mu \mathrm{m} / \mathrm{voxel}$ and assuming that the unloading at $65 \%$ of the maximal load is elastic, it means that the final crack opening were approximately $24 \mu \mathrm{m}$ near the notch extremity.

It has been checked that the displacement field amplitudes are proportional to the load for the first scans within the elastic domain before the crack initiation.

The overall kinematic of the double-torsion test is well measured, as illustrated by the deformed meshes (Fig. 7). From one load step to another, the deformation is
Fig. 4 XCT scan $n^{\circ} 8$ of the zone located just above the initial notch on the side loaded in tension $(1 \operatorname{voxel}=7.9 \mu \mathrm{m})$

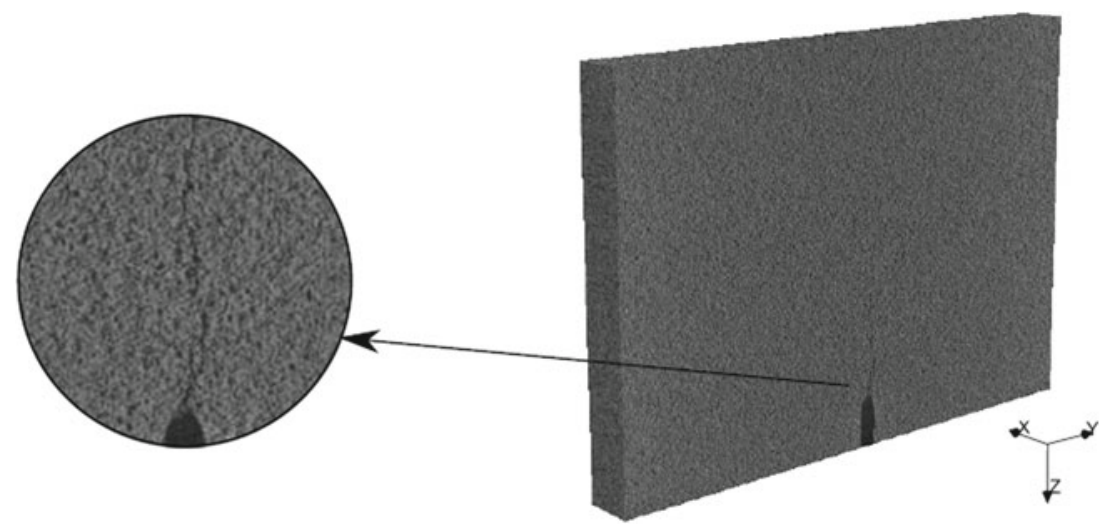




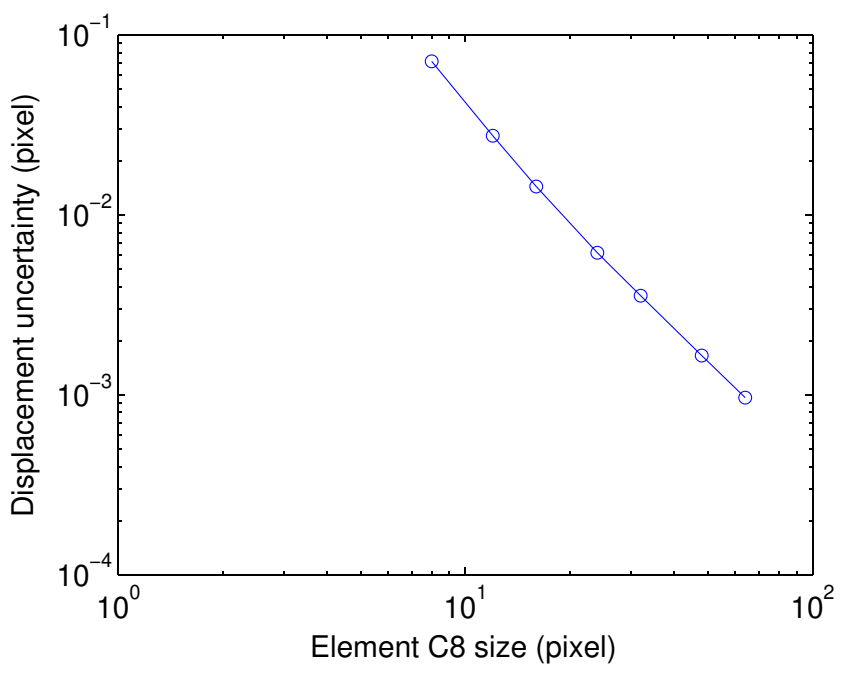

Fig. 5 Theoretical displacement uncertainties depending on the mesh used for the XCT scans

symmetric with respect to the crack plane. This is consistent with the usual hypothesis which consists in considering the two cracked half-parts as a succession of twisted beams [1, 14]. After the loading up to $8.5 \mathrm{~N}$, it can be seen on Fig. 7 corresponding to the scan $n^{\circ} 2$, that the sample is no more perfectly normal to the X-ray beam axis, but has slightly turned of an angle of 30 degrees, probably due to plays in the test rig. DVC enables to detect this rotation and to correct the measured displacement fields.
The strain field is nevertheless quite noisy due to the low displacement amplitude. Indeed, the measured strains, between $\pm 0.5 \%$ in the direction $y$, are here already six times greater than the known fracture strain of this ceramic. The accuracy is sufficient to visualize the crack location, but not enough to check for exemple, the first DT assumption which states that the uncracked part of the specimen remains undeformable. To obtain more reliable quantitative strain values, different solutions can be considered:

- Increasing the element size to 64 voxels would improve the measurement accuracy and lead to less noisy strain fields. But it would reduce too much the number of elements in the sample thickness (Fig. 6).

- Increasing the spatial resolution. The displacements measured in voxels would be larger so that the derived strains would be less noisy. But it would also significantly reduce the size of the observed zone and the opportunity to observe the crack tip propagation at different load steps. This approach was nevertheless tested on another sample for which two scans of two different resolutions have been acquired at each load step. A very good agreement between the different fields obtained from the two scans at the same load step is observed (Fig. 8). As expected, the strain field with the best spatial resolution is less noisy but the observed zone becomes too small.
Fig. 6 Influence of the mesh size on the displacement $V_{y}$ measured by our DVC method during a double-torsion test $(1$ voxel $=9.3 \mu \mathrm{m})$ (a)

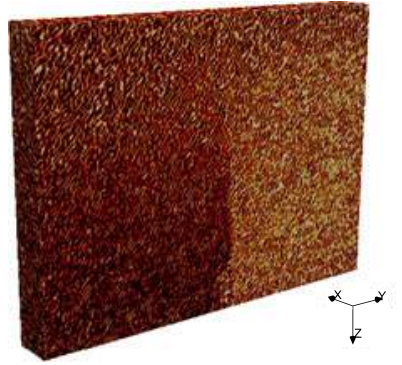

Elements $\mathrm{C} 8=8$ voxels

(c)

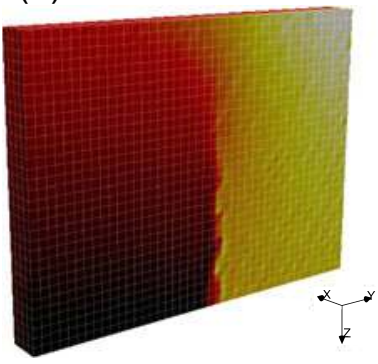

Elements C8 $=32$ voxels

(b)
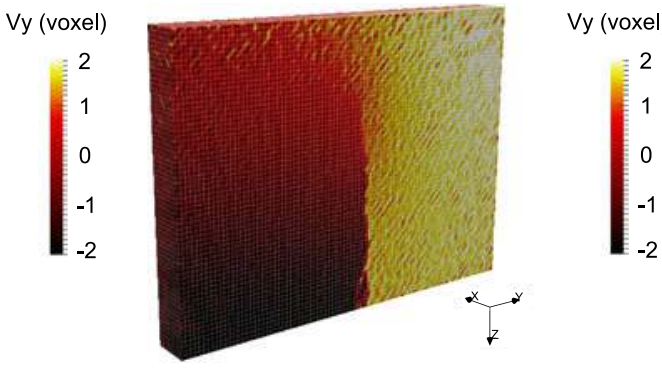

Elements C8 $=16$ voxels

(d)
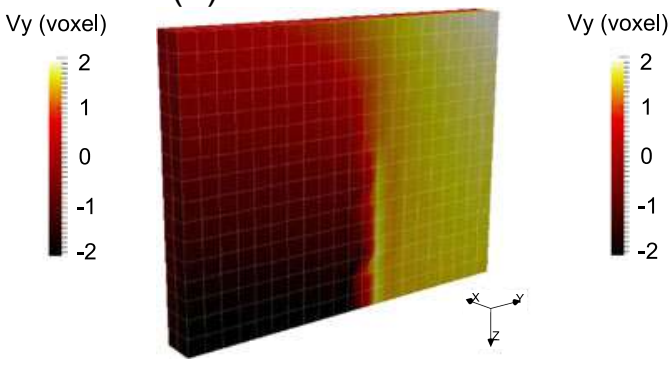

Elements C8 $=64$ voxels 
(a)
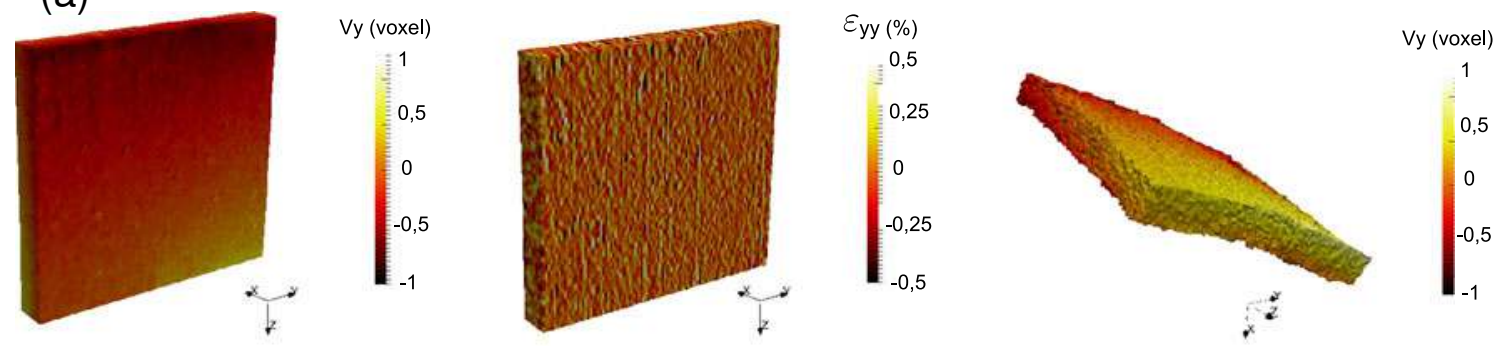

scan $n^{\circ} 2$ (view of the face in tension)

(b)
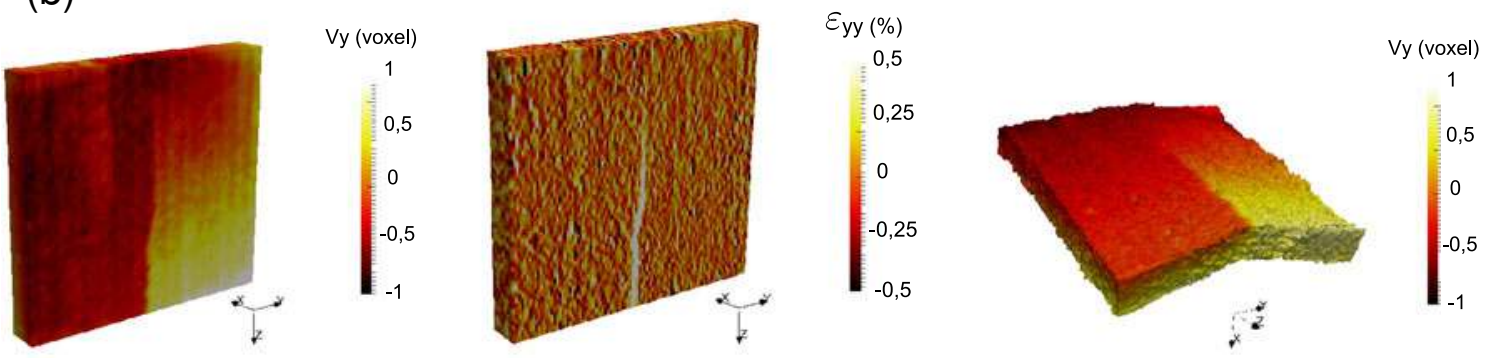

scan $n^{\circ} 5$ (view of the face in tension)

(c)
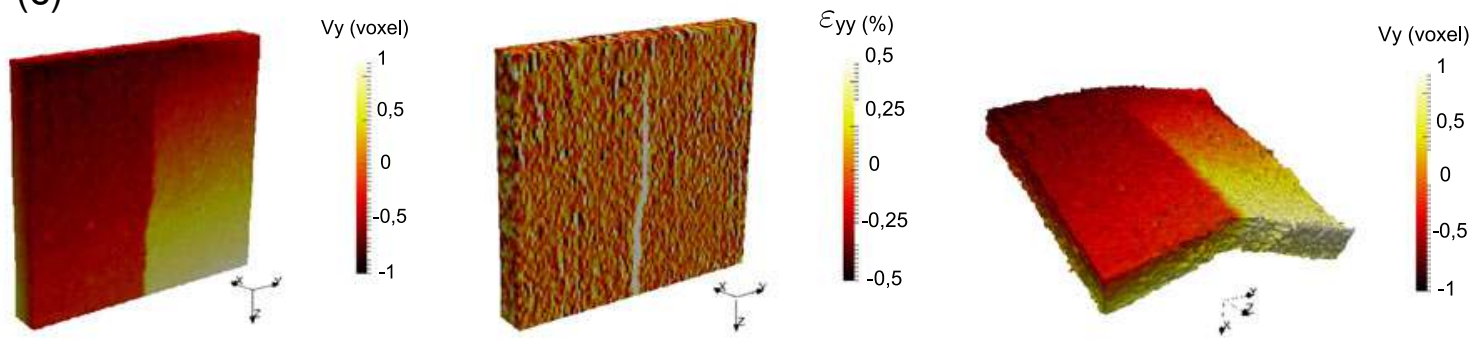

scan $n^{\circ} 6$ (view of the face in tension)

(d)
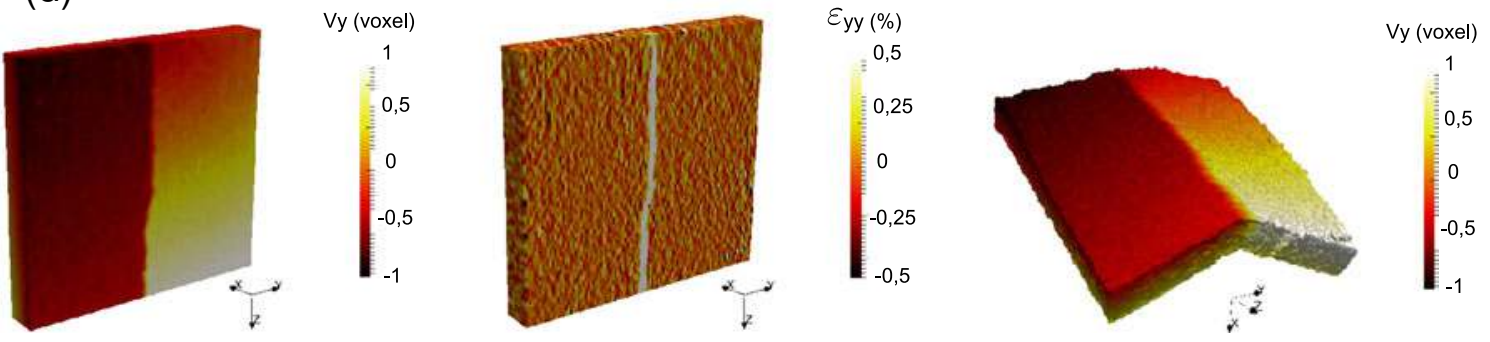

scan $n^{\circ} 7$ (view of the face in tension)

Fig. 7 Evolution of the displacement field $V_{y}$ (left), the stain field $\epsilon_{y y}$ (center) and the deformed mesh with a $\times 100$ amplitude $($ right) for four different scans of the silicon carbide sample loaded in double-torsion (Elements $\mathrm{C} 8=16$ voxels, 1 voxel $=7.9 \mu \mathrm{m}$ )

- Performing the scans without unloading at $65 \%$ of the maximal load. This would increase the measured relative displacements and this could be achieved without modifying the spatial resolution and the element size. Nevertheless, a more powerful XCT scanner is then required to perform a full scan as fast as possible [34] to avoid or limit slow crack growth during the scan duration.

These problematics about the accuracy are classical for fullfield measurement methods but are here exacerbated by the low deformation of the tested ceramic. The range of 
Fig. 8 Superposition of displacement $V_{y}(l e f t)$ and strain $\epsilon_{y y}$ (right) fields with two spatial resolutions (Elements $\mathrm{C} 8=16$ voxels, 1 voxel $=9.3 \mu \mathrm{m}$ and Elements $\mathrm{C} 8=32$ voxels, 1 voxel $=3.1 \mu \mathrm{m}$ ) - The displacement scale of the scan with the finest resolution has been divided by three to keep the color equivalence
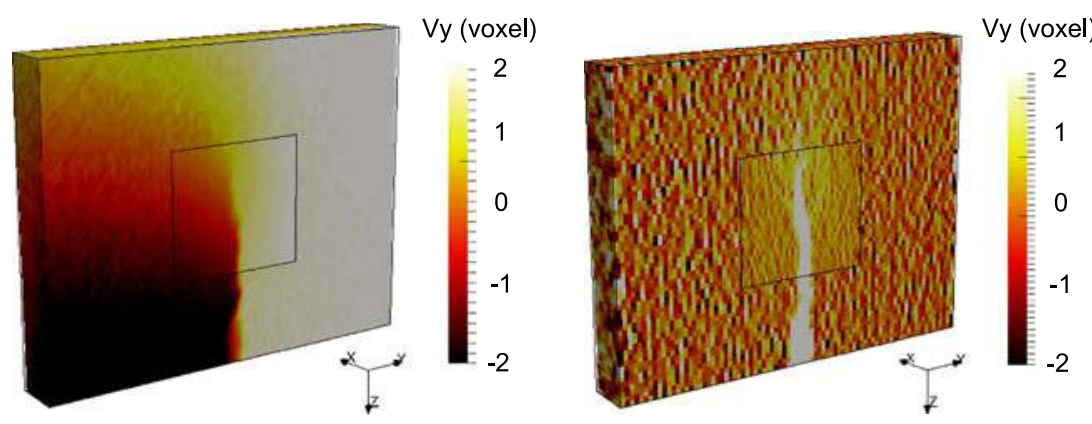

material analysed using DVC includes materials with higher deformability, such as foams [22] or cast iron [23] so that the measured strain amplitudes are much higher.

\section{Crack Front Detection in 3D}

A strong discontinuity of the displacement field $V_{y}$ is shown in Fig. 7, revealing the crack propagation along the vertical axis. The crack path is clearly visible on the tensile face, whereas it is not on the compressive face (Fig. 9). It means that the crack front does not reach the compressive face. This result is consistent with previous observations in the literature concerning the asymmetrical shape of the crack front in DT $[15,16]$.

The DVC technique enables the detection of the crack path (equation (6)) through the use of the correlation residual $R$. Indeed, since the $\mathrm{C} 8$ finite element shape functions assume a continuous displacement field, the solution $\mathbf{v}$ can not respect the displacement discontinuity generated by the crack. Whereas the mean value is low (about $2 \%$ ), the correlation residual $R$ is much higher along the crack and this allows an easier detection of the crack location. Figure 10 represents the correlation residual over the tensile face near the crack for the scan $n^{\circ} 6$. As expected, the crack path over
Fig. 9 Displacement $V_{y}$ (left) and strain $\epsilon_{y y}$ (right) fields. Scan $n^{\circ} 6$ of the DT test. Tensile and compressive faces of the sample (Elements $\mathrm{C} 8=16$ voxels, 1 voxel $=7.9 \mu \mathrm{m}$ )
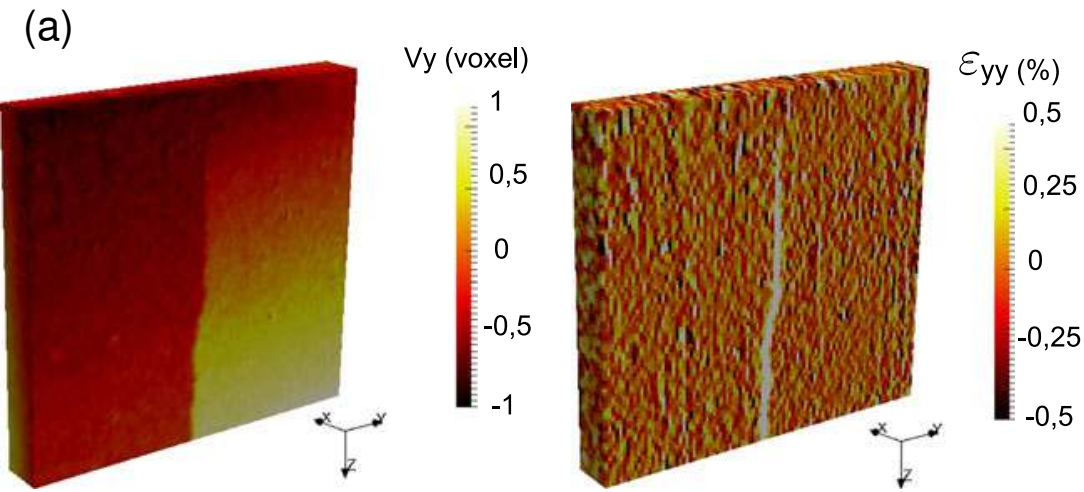

scan $n^{\circ} 6$ (view of the tensile face of the specimen) (b)
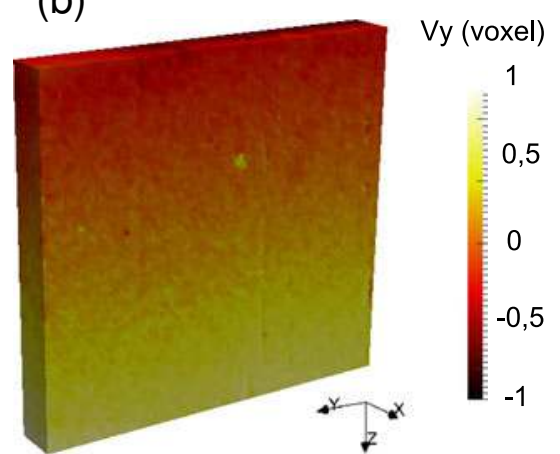

$\varepsilon_{\mathrm{yy}(\%)}$

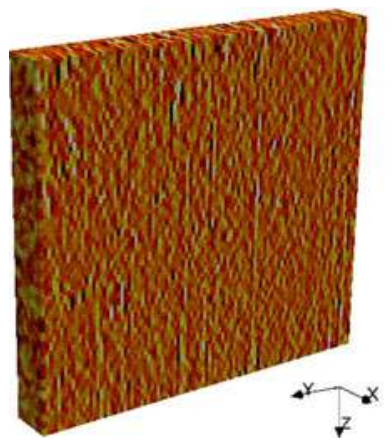
men)

scan $n^{\circ} 6$ (view of the compressive face of the speci- 


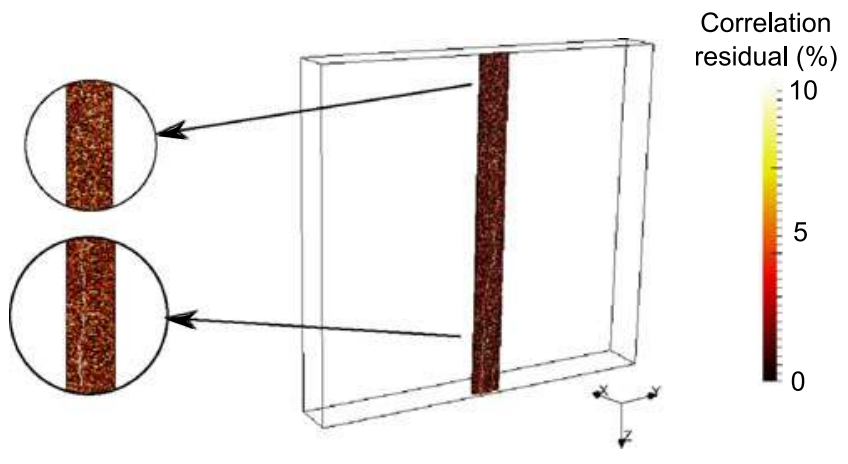

Fig. 10 Correlation residual $R$ for the scan $n^{\circ} 6$ on the silicon carbide sample (Elements $\mathrm{C} 8=16$ voxels, 1 voxel $=7.9 \mu \mathrm{m}$ )

this face is clearly visible. But unfortunately, it is possible to locate exactly neither the crack tip, not the crack front through the sample thickness. One reason for that may be the very high porosity of this ceramic.

To detect the crack front location, a threshold is applied to the strain field to keep only the elements with a very high deformation along $y$ direction. A threshold value of $\epsilon_{y y}>7 \cdot 10^{-3}$ is here arbitrarily chosen. This strain value has no physical meaning but enables to detect the crack front at each load step (Fig. 11). It has been checked that varying this threshold value from $\pm 30 \%$ does not change significantly the crack front profiles (Fig. 12). The same approach has been used for DIC and DT in 2D [37]. DVC allows to estimate a crack length of $4.1 \mathrm{~mm}$ over the tensile face between the scans $n^{\circ} 4$ and $n^{\circ} 5$, and of $2.5 \mathrm{~mm}$ between the scans $n^{\circ} 6$ and $n^{\circ}$. The uncertainty on these propagation distances is about $\pm 0.5 \mathrm{~mm}$. The crack propagation velocities are difficult to quantify since the unloading and the loading were too slow between $65 \%$ and $100 \%$ of the nominal load.

Thanks to this alternative strain threshold approach, it is possible to follow the evolution of the crack front (Fig. 11). The front is highly curvilinear. It comes from the fact that the propagation in mode I occurs only in tension. This detected curvilinear shape is consistent with observations carried out on transparent materials [16] and with numerical simulations where a quarter-ellipse front was chosen [19]. Even for the very last load step before the sudden fracture of the sample, the crack front has not yet reached the compressive side of the specimen. This remaining

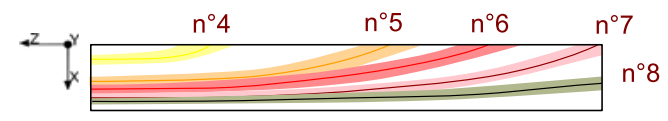

Fig. 12 Crack front detection uncertainties for a DT specimen analysed using DVC (Elements C8 $=16$ voxels, 1 voxel $=7.9 \mu \mathrm{m}$ )

ligament contributes probably to stabilize the crack propagation by reducing the stress intensity factors as already suggested [3].

\section{Conclusion and Prospects}

Double-torsion is a widely used test in the literature to characterize the fracture behavior of ceramic materials. Its interpretation is based on the assumptions of linear elasticity and on some simplified considerations regarding the specimen behaviour. It leads, at first approximation, to a straight crack front and a stress intensity factor $K_{I}$ independent of the crack length. These hypotheses, already questioned in the literature, could be still refined through the understanding brought by an in situ DT test analysed by XCT and DVC techniques.

A specific experimental DT set-up to perform in situ tests in a laboratory tomograph was specially designed. DVC was used to monitor the three-dimensional displacement field within the opaque samples during the stable crack growth propagation. The overall kinematics was successfully captured. The evolution of the crack front during the propagation was identified using a threshold on the strain field. It allowed to confirm that the crack fronts are highly curvilinear and do not reach the sample face loaded in compression even for an important crack propagation length.

Because of some material specificities (very high porosity and low failure strain), the present measurement quality is however not fully sufficient to be able to check all the DT hypotheses. That's why some improvements are proposed to still increase the accuracy:

- Using a faster acquisition procedure to have very short acquisition times. The objective is to avoid the unloading of the sample at $65 \%$ of the maximal load which
Fig. 11 Threshold on the strain $\epsilon_{y y}$ field to detect the 3D crack front evolution (Elements $\mathrm{C} 8=$ 16 voxels, 1 voxel $=7.9 \mu \mathrm{m}$ )

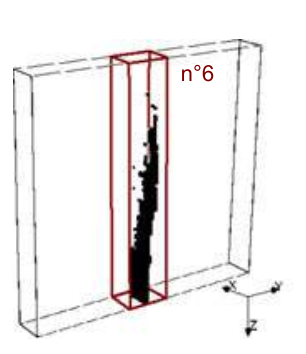

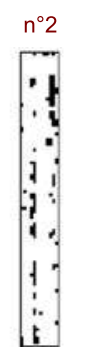
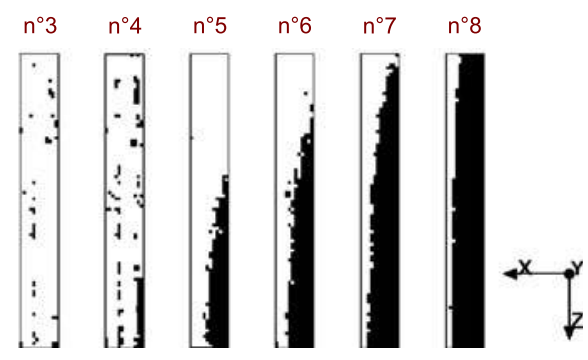
decreases the measured relative displacement amplitude. In principle, it has even been demonstrated using synchrotron tomographs [34] that a continuous loading experiment could be performed. This would be extremely valuable for the present problem.

- Stitching several scans taken at lower resolutions. The advantage would be to improve the accuracy without shrinking the zone where the crack front can be detected.

- Developing some specific shape functions for DIC to decompose the displacement field over the area of interest dedicated to DT kinematics to improve the robustness of the displacement estimation as performed previously [28-31].

Thanks to these improvements, the next possible steps will consist, amongst others, in using these experimental data for numerical simulations to determine the stress intensity factor along the actual crack front during a slow crack growth propagation. It will enable us to study in detail the common hypothesis about the constant value of $K_{I}$ versus the crack length.

This approach represents a promising way to analyse in detail and to improve our understanding of complex tests, such as the double-torsion one, for brittle materials.

Acknowledgments This work is financially supported by the ANRT (National Association for Research and Technology) through the contract $n^{\circ} 2008 / 501$. The authors wish to thank Fabiano Rodrigues and his colleagues at the CREE (Saint-Gobain group) for providing the samples and supporting this research project.

\section{References}

1. Evans AG (1973) A simple method for studying slow crack growth in brittle materials. Int J Fract 9(3):267-275

2. Shyam A, Lara-Curzio E (2006) The double-torsion testing technique for determination of fracture toughness and slow crack growth behavior of materials: a review. J Mater Sci 41(13): 4093-4104

3. Chevalier J (1996) Etude de la propagation des fissures dans une zircone 3 Y-TZP pour applications biomedicales. $\mathrm{PhD}$ thesis, INSA de Lyon

4. Rhanim H, Olagnon C, Fantozzi G, Torrecillas R (1997) Crack propagation behaviour in mullite at high temperatures by doubletorsion technique. J Eur Ceram Soc 17(1):85-89

5. Outwater JO, Gerry DJ (1969) On the fracture energy, rehealing velocity and refracture energy of cast epoxy resin. J Adhes 1(4):290-298

6. Evans AG, Wiederhorn SM (1974) Crack propagation and failure prediction in silicon nitride at elevated temperatures. J Mater Sci 9(2):270-278

7. Quinn GD (1987) Delayed failure of a commercial vitreous bonded alumina. J Mater Sci 22(7):2309-2318

8. Gremillard L, Epicier T, Chevalier J, Fantozzi G (2000) Microstructural study of silica-doped zirconia ceramics. Acta Mater 48(18-19):4647-4652
9. Baskaran S, Bhaduri SB, Hasselman DPH (1985) Effect of crystallites on subcritical crack growth and strain-rate sensitivity of strength of cordierite glass-ceramics. J Am Ceram Soc 68(3): 112-119

10. Ciccotti M (2000) Realistic finite element model for the double-torsion loading configuration. J Am Ceram Soc 83(11): 2737-2744

11. Shyam A, Lara-Curzio E (2008) Mechanical characterization of diesel particulate filter substrates. Am Ceram Soc 6: 1995-2001

12. Fuller ER (1979) An evaluation of double-torsion testing-analysis Fracture Mechanics Applied to Brittle Materials. ASTM International

13. Tait RB, Fry PR, Garrett GG (1987) Review and evaluation of the double-torsion technique for fracture toughness and fatigue testing of brittle materials. Exp Mech 27(1):14-22

14. Boussuge M (1985) Contribution a l'etude du comportement mecanique a haute temperature du nitrure et du carbure de silicium: Utilisation critique de l'essai de double-torsion. $\mathrm{PhD}$ thesis, Ecole Nationale Superieure des Mines de Paris

15. Leevers PS (1982) Crack-front shape effects in the double torsion test. J Mater Sci 17(9):2469-2480

16. Frassine R, Riccò T, Rink M, Pavan A (1988) An evaluation of double-torsion testing of polymers by visualization and recording of curved crack growth. J Mater Sci 23(11):40274036

17. Pollet JC, Burns SJ (1979) Crack-velocity correction factor for the crack-front shape in double torsion specimens. J Am Ceram Soc 62(7-8):426-427

18. Trantina GG (1977) Stress analysis of the double-torsion specimen. J Am Ceram Soc 60(7-8):338-341

19. Tseng AA, Berry JT (1979) A three-dimensional finite element analysis of the double-torsion test. J Press Vessel Technol $101: 328$

20. Ciccotti M, Gonzato G, Mulargia F (2000) The double torsion loading configuration for fracture propagation: An improved methodology for the load-relaxation at constant displacement. Int J Rock Mech Min Sci 37(7):1103-1113

21. Buffière JY, Maire E, Cloetens P, Lormand G, Fougères R (1999) Characterization of internal damage in a $\mathrm{mmc}_{i} \mathrm{sub}_{\dot{i}} \mathrm{p}_{i} / \mathrm{sub}_{\dot{\zeta}}$ using $\mathrm{x}$-ray synchrotron phase contrast microtomography. Acta Mater 47(5):1613-1625

22. Roux S, Hild F, Viot P, Bernard D (2008) Three-dimensional image correlation from X-ray computed tomography of solid foam. Compos A: Appl Sci Manuf 39(8):1253-1265

23. Rannou J, Limodin N, Réthoré J, Gravouil A, Ludwig W, BaiettoDubourg M-C, Buffiere J-Y, Combescure A, Hild F, Roux S (2010) Three dimensional experimental and numerical multiscale analysis of a fatigue crack. Comput Methods Appl Mech Eng 199:1307-1325

24. Buffiere JY, Maire E, Adrien J, Masse JP, Boller E (2010) In situ experiments with $\mathrm{x}$ ray tomography: An attractive tool for experimental mechanics. Exp Mech 50(3):289-305

25. Parkinson DY, Cox BN, Marshall DB, Ritchie RO (2012) Real-time quantitative imaging of failure events in materials under load at temperatures above $1600 \mathrm{c}$. Nat Mater. doi:10.1038/NMAT3497

26. Sutton MA, Wolters WJ, Peters WH, Ranson WF, McNeill SR (1983) Determination of displacements using an improved digital correlation method. Image Vision Comput 1(3):133139

27. Besnard G, Hild F, Roux S (2006) Finite-element displacement fields analysis from digital images: Application to portevin-le châtelier bands. Exp Mech 46(6):789-803 
28. Roux S, Hild F (2006) Stress intensity factor measurements from digital image correlation: Post-processing and integrated approaches. Int J Fract 140(1):141-157

29. Leplay P, Réthoré J, Meille S, Baietto M-C (2011) Identification of damage and cracking behaviours based on energy dissipation mode analysis in a quasi-brittle material using digital image correlation. Int J Fract 171(1):35-50

30. Leplay P, Réthoré J, Meille S, Baietto M-C (2010) Damage law identification of a quasi-brittle ceramic from a bending test using digital image correlation. J Eur Ceram Soc 30: 2715-2725

31. Hild F, Roux S (2006) Digital image correlation: From displacement measurement to identification of elastic properties-a review. Strain 42(2):69-80

32. Leplay P, Réthoré J, Meille S, Baietto MC (2012) Identification of asymmetric constitutive laws at high temperature based on digital image correlation. J Eur Ceram Soc 32(15):3949-3958
33. Bay BK, Smith TS, Fyhrie DP, Saad M (1999) Digital volume correlation: Three-dimensional strain mapping using X-ray tomography. Exp Mech 39(3):217-226

34. Maire E, Carmona V, Courbon J, Ludwig W (2007) Fast x-ray tomography and acoustic emission study of damage in metals during continuous tensile tests. Acta Mater 55(20):68066815

35. Badel P, Vidal-Sallé E, Maire E, Boisse P (2008) Simulation and tomography analysis of textile composite reinforcement deformation at the mesoscopic scale. Compos Sci Technol 68(12): 2433-2440

36. Chevalier J, Saadaoui M, Olagnon C, Fantozzi G (1996) Double-torsion testing a 3y-tzp ceramic. Ceram Int 22(2):171177

37. Becker TH, Marrow TJ, Tait RB (2011) Damage, crack growth and fracture characteristics of nuclear grade graphite using the Double Torsion technique. J Nucl Mater 414(1):32-43 